\title{
AHP for Comprehensive Approach of Modern QFD
}

\begin{abstract}
The aim of the paper is to explain why and illustrate how the practice of Comprehensive QFD is enhanced with incorporating AHP. With AHP, QFD could use valid priority data to perform coherent and cohesive deployment. With AHP, QFD is able to address with greater the needs of not a single but multiple customers and seamlessly link the project with management goals. To explain how AHP is used in Comprehensive QFD in greater detail, a reported case about an energy transition programme was borrowed for illustration.
\end{abstract}

Keywords: Quality Function Deployment, Comprehensive QFD, Modern QFD, AHP, ISO 16355, Blitz QFD ${ }^{\circledR}$.

\section{Introduction}

Analytic hierarchy process (AHP) enhances the powerfulness of quality function deployment (QFD). Mastered with a skillful manipulation of the quality management principles, QFD was first suggested in the mid-1960s in Japan as an operation mechanism for practicing Hoshin Kanri and doing product development. QFD provides an effective process for teaming up organizational members from various departments and different levels. Guided by the system and using the tools and methods suggested by QFD, team members have their efforts aligned to specify the functional requirements and devise plans in regard to the needs of the customers. QFD reached the USA in the early 1980s. Although organizations were aware of the benefits of QFD, the long time required for completing the whole matrix of matrices and the generation of inaccurate priority data had greatly hindered the full application of QFD in industries. Fortunately, after a few years of studies of the QFD experts, the formulation of Blitz QFD $^{\circledR}$ and the introduction of AHP helped overcome the two shortcomings (Mazur, 2015).

The incorporation of AHP enables QFD to be practiced in a valid and comprehensive way. Although AHP has been used in QFD for more than two decades, some applications remain partial. In many cases, AHP was only used to prioritize the demanded qualities and identify the important quality elements. However, the real benefits of AHP to QFD are far more than this. AHP powers the operation of QFD by supplementing with the essential mechanism for quantification. With the added power, QFD could be readily applied to projects of much larger scale and used to address a wider range of aspects with each aspect of greater depth. The aim of the paper is to explain why and illustrate how the practice of Comprehensive QFD is enhanced with incorporating AHP, with using the case about the programme of "Transition to Low Carbon Dioxide Energy System" reported by Stansfield, Colechin and Mazur in 2016 for illustration.

\section{AHP for QFD}


Although AHP is quantitative and QFD is qualitative in nature, they both use hierarchical structures to display their models and use them as the backbone for operation. The relationship of dependency and the stratification by levels of hierarchy as well as focusing on the vital few are the key ideas that are in common to both of them. As a model for decision making, AHP is formulated in such way that the derived priorities could give a proportionate ordering of the different possible outcomes to which one can allocate resources in an optimal way (Saaty, 2007). The excellent mathematical formulation and user-friendly operation of AHP supplements QFD. Below are four main features of the outstanding model of AHP that enhance the operation of QFD:

\subsection{AHP uses ratio scale to collect responses}

In Classical QFD, respondents were commonly asked to rank a set of given needs in numerical order to prioritize their importance and use a set of ordinal numbers to indicate the strength of the relationship between two items. However, the numbers collected by such survey methods could hardly produce valid mathematical meaning. AHP uses ratio scale to collect responses. As responses in ratio scale could be put into addition, subtraction, multiplication and division, therefore judgements from a group of respondents could be combined and team decision could also be facilitated.

\subsection{AHP uses pairwise comparison to capture judgments}

People could not always give precise judgments which resulted with receiving responses that are not actual and exact. The pairwise comparison of AHP helps QFD on receiving quality responses. For a given set of needs, criteria or alternatives, the respondent is asked to make comparison between any two of them at a time on the importance with respect to the specified goals and continue to compare until every pair has been judged. To collect responses in such a thorough way and with a further check on the consistency of the responses, not only accurate judgments could be received but rankings and magnitudes of the judgments are also informed.

\subsection{AHP uses ratio numbers to present priorities}

AHP yields outputs in ratio numbers which is particularly important to the comprehensive approach of QFD. As ratio numbers are mathematically operative, they could be deployed from high-level system design to detailed components and processes. Priority data could be transferred from one matrix to another matrix with high accuracy.

\subsection{AHP helps focusing on important branches}

AHP turbocharges Blitz QFD ${ }^{\circledR}$. Instead of making deployment one time one matrix to one matrix as was the way of Classical QFD, the maximum value table of Blitz QFD ${ }^{\circledR}$ speeds up the deployment process by translating the customer needs into elements of all the aspects constituting the solution in one table. Coupled with AHP, the efficiency of Blitz $\mathrm{QFD}^{\circledR}$ is further raised. Putting the items in a hierarchical structure, applying the topdown approach and focusing on the high-value branches, the most important items could be determined without the need of evaluating all the items.

\section{Comprehensive Approach of Modern QFD}

To explain how the mentioned features of AHP enhance the comprehensive approach of Modern QFD, let us take a look to the case about the programme of "Transition to Low 
Carbon Dioxide Energy System", which was reported by Stansfield, Colechin and Mazur in 2016.

\subsection{Align QFD project with business goals}

Organizations could hardly succeed if they could not map out how their projects would contribute to achieving the business goals. The first, and also the most important, step of Modern QFD is to align the project with business needs. For the case of the energy transition programme, the team members started the project with exploring and identifying those outcomes and goals that were of greatest strategic value to the organization so as to clarify what the programme would contribute and what should be achieved by the programme. In regard to the government requirements of new energy systems, the team members discussed, interpreted and turned the government requirements, which were the business drivers or the external outcomes of the project, into organizational outcomes, and, further deployed the organizational outcomes into programme goals. Upon displaying their deployments in the form of a hierarchy diagram, an AHP exercise was conducted to establish the relative importance for each of the outcomes and the goals (Figure 1). The generated priorities were going to be transferred to the subsequent steps of the project for planning the programme.

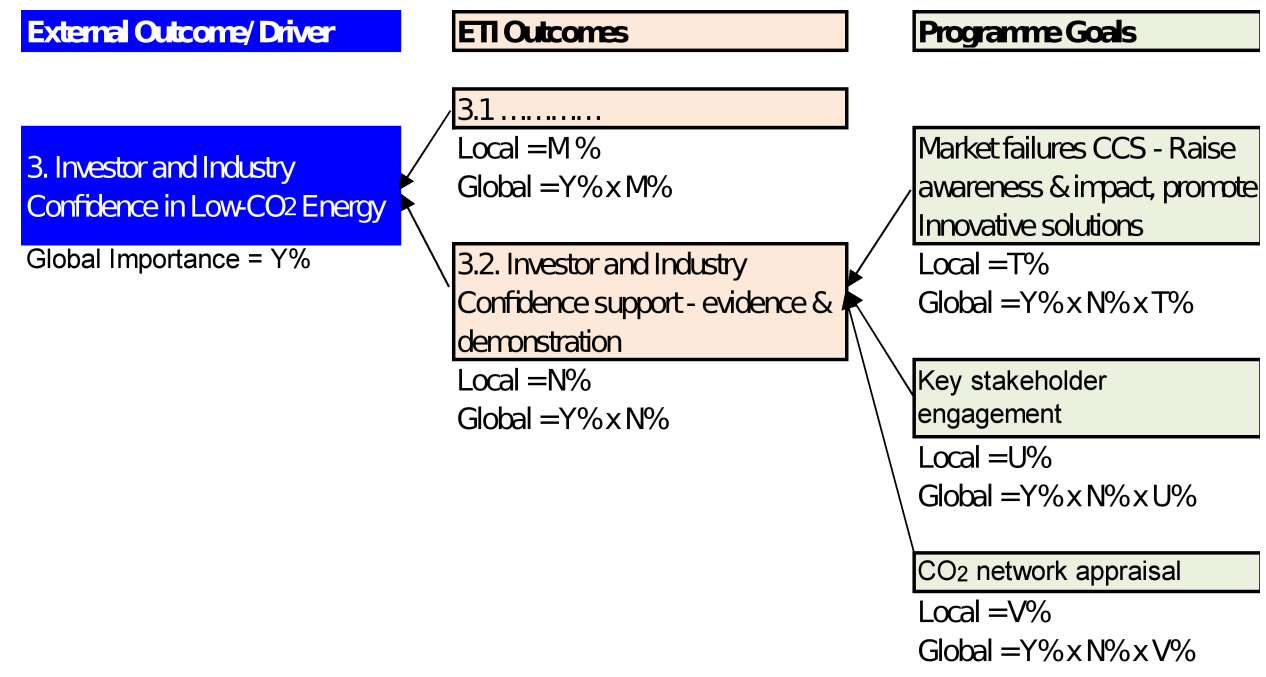

Figure 1: Hierarchy Diagram of Outcomes and Goals of the Energy Transition Programme

\subsection{Review stakeholder impacts}

Due to the extremely complex supply chain of the energy industry, the transition to an affordable, secure and low carbon dioxide energy system was not simply a matter between the supplier and the users. Understanding that they needed to carefully review the gain and the loss of the players and properly address the needs of the key stakeholders, the team members continued the project with doing an exercise on stakeholder prioritization. They first used the method of "Supplier, Input, Process, Output, Customer (SIPOC)" mapping to identify the key stakeholders and then with a QFD matrix to assess potential impact of each stakeholder on successful delivery of each programme goal with the use of a normalized scale. With importing the relative 
importance of the programme goals that obtained from the previous AHP exercise, the generated data helped the team establish actions for clarifying the needs of the high priority stakeholders.

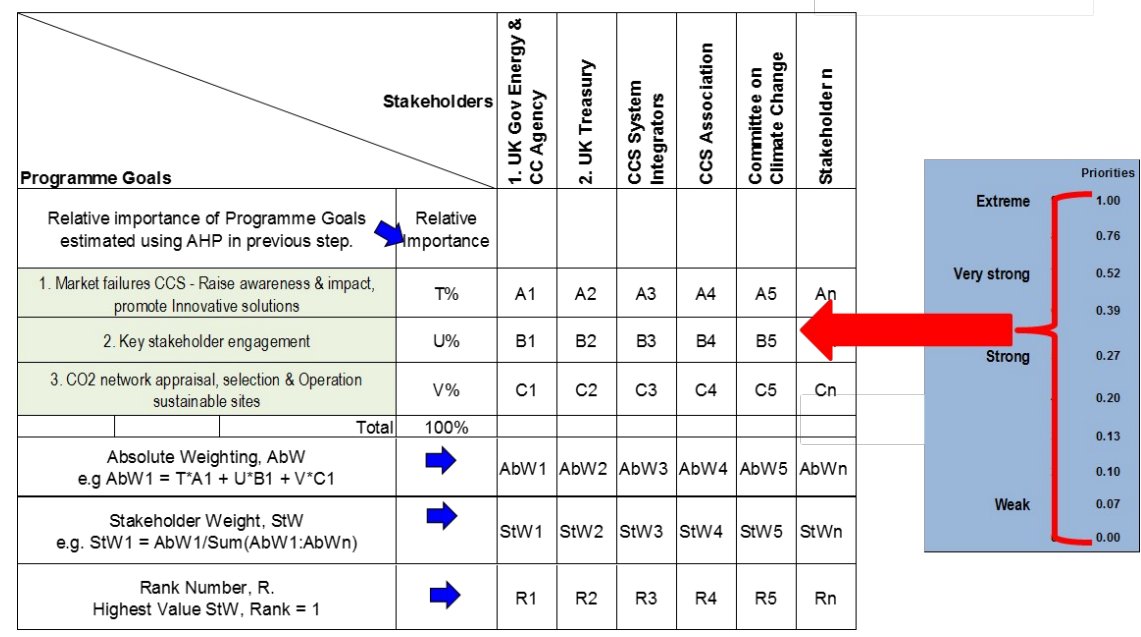

Figure 2: An Excerpt of the QFD Matrix for Assessing Impact of Stakeholders on Programme Goals of the Energy Transition Programme

\subsection{Link customer needs to requirements for solution design}

Upon collected the voice of the key stakeholders, the team members of the energy transition programme used a table with two sides to assist them on doing the deployment. They first interpreted the collected voice into the stakeholder needs and recorded down the items they had extracted from their analysis on the left hand side of the table, that is, the Customer Voice Table (CVT). The team members continued the deployment with translating the stakeholder needs into the requirements of solution design. They put down the generated ideas and suggested items on the right hand side of the table, that is, the Maximum Value Table (MVT).
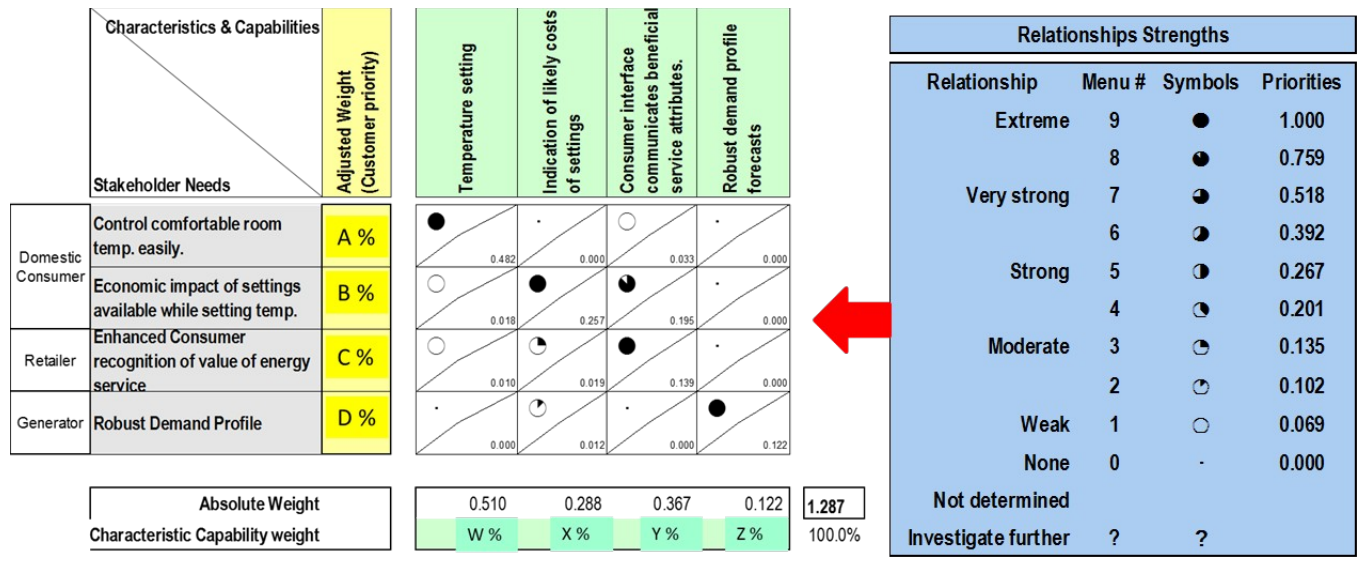

Figure 3: An Excerpt of a QFD matrix for Assessing Solution Elements against

\section{Stakeholder Needs}

International Symposium on the 
For the customer side, members of the organization who had worked closely with the stakeholders, though ideally was representatives of the key stakeholders, were invited to come to do an AHP exercise on seeking the relative importance of each of the needs. For the solution side, the project team focused on identifying the high-value requirements for meeting the needs. For each of the aspects stated on the MVT, the team members used a QFD matrix to assess the relationship between the elements of the aspect and the needs of the stakeholders with a normalized scale (Figure 3). After completed the assessment of all the aspects, the high value requirements were found. These requirements would be those on which design efforts could be focused.

\subsection{Evaluate design options}

The QFD project continued. After completed the development of design options for addressing the high value requirements, once again, the team incorporated AHP into Pugh concept selection to evaluate the design options.

\section{Conclusion}

Using ratio scale to collect responses and pairwise comparison to capture judgments, presenting priority data in ratio numbers as well as making use of top-down approach to locate the important branches are the key features of AHP that supplement QFD. With AHP, QFD is able to perform in a more comprehensive way, including properly aligning the project with management goals, conducting all-round review with the needs of the stakeholders, efficiently and effectively linking the customer needs to the requirements for solution design as well as rationally selecting the optimal design.

The new ISO 16355 International Standard for QFD was published in 2017. It is a "framework" based on Yoji Akao's Comprehensive QFD Model and the standard includes guidance in its application to products and services of various industries. An expert guidance provided by the standard is the use of AHP to quantify both the customer needs and relationship weights. As Mazur (2017) explained, the improved accuracy of using ratio scale values derived with AHP will be helpful to the transfer of prioritization - a very critical part of QFD.

\section{Key References}

Mazur, G. (2015). ISO 16355 - The international standard for QFD. The Paper Collection of the 21 International Symposium on Quality Function Deployment, 10-21.

Mazur, G. (2017). Transition from JIS Q 9025 to ISO 16355. The $23^{\text {rd }}$ International Symposium on Quality Function Deployment, 20-42.

Saaty, T.L. (2007). The analytic hierarchy process: How to measure intangibles in a meaningful way side by side with tangibles. Transactions from The International Symposium on QFD 2007-Williamsburg \& The $19^{\text {th }}$ Symposium on Quality Function Deployment, 113-135.

Stansfield, K., Colechin, M., \& Mazur, G. H. (2016). Soft systems method integration with sustainable energy systems development using ISO 16355 for QFD. Transactions 
ISAHP Article: A Style Guide for Paper Proposals To Be Submitted to the International Symposium on the Analytic Hierarchy Process 2018, Hong Kong, HK.

from The $22^{\text {nd }}$ International \& The $27^{\text {th }}$ North American Symposium on Quality Function Deployment, 14-39. 